\title{
Acometimento vascular na doença de Behçet: o processo imunopatológico
}

\author{
Vascular involvement in Behçet's disease: the immunopathological process \\ Raquelle Machado de Vargas', Maria Luiza Nunes da Cruz'1, Maria Paula Hashimoto Giarllarielli', Beatriz Mota Sano, \\ Geovana Idelfoncio da Silva', Karina Furlani Zoccal', Cristiane Tefé-Silva' ${ }^{1}$ (D)
}

\begin{abstract}
Resumo
A doença de Behçet constitui uma forma rara de vasculite sistêmica, que acomete de pequenos a grandes vasos. É caracterizada por manifestações mucocutâneas, pulmonares, cardiovasculares, gastrointestinais e neurológicas. Sua apresentação clínica é bastante ampla, variando de casos mais brandos a casos graves, com acometimento multissistêmico, caracteristicamente com exacerbações e remissões. Suas causas ainda são desconhecidas; entretanto, há evidências genéticas, ambientais e imunológicas, como a associação com o alelo HLA-B51. Todas essas, em conjunto, apontam para um processo imunopatológico anormal, com ativação de células da imunidade inata e adaptativa, como as células natural killer, neutrófilos e células T, que geram padrões de respostas e citocinas específicos capazes de gerar mediadores que podem lesionar e inflamar o sistema vascular, resultando em oclusões venosas, arteriais e/ou formação de aneurismas.
\end{abstract}

Palavras-chave: síndrome de Behçet; antígeno HLA-B51; vasculite sistêmica.

\begin{abstract}
Behçet's disease is a rare form of systemic vasculitis that affects small to large vessels. It is characterized by mucocutaneous, pulmonary, cardiovascular, gastrointestinal, and neurological manifestations. Its clinical presentation is quite wide, ranging from milder cases to severe cases, with multisystemic involvement, characteristically with exacerbations and remissions. Its etiopathogenesis is still unclear, although there is evidence of genetic, environmental, and immunological factors, such as the association with the HLA-B51 allele. In conjunction, all of these point to an abnormal immunopathological process, with activation of cells of innate and adaptive immunity, such as NK cells, neutrophils, and T cells, which generate specific response patterns and cytokines capable of generating mediators that can damage and inflame blood vessels, resulting in venous and arterial occlusions and/or aneurysm formation.
\end{abstract}

Keywords: Behçet syndrome; HLA-B51 antigen; systemic vasculitis.

Como citar: Vargas RM, Cruz MLN, Giarllarielli MPH, et al. Acometimento vascular na doença de Behçet: o processo imunopatológico. J Vasc Bras. 2021;20:e20200170. https://doi.org/10.1590/1677-5449.200170 


\section{INTRODUÇÃO}

A doença de Behçet (DB) é uma síndrome inflamatória complexa, multissistêmica, crônica e de etiologia desconhecida, descrita inicialmente pelo dermatologista turco Hulusi Behçet em 1937. Do ponto de vista clínico, é uma vasculite que apresenta vários fenótipos, acometendo pequenos a grandes vasos, caracterizada por úlceras orais recorrentes e outras manifestações, que incluem afecções pulmonares, cardiovasculares, gastrointestinais e neurológicas ${ }^{1}$. A DB tem distribuição mundial, mas a maioria dos casos é encontrada na chamada "rota da seda", a qual se estende da Ásia ao Mediterrâneo ${ }^{1}$. A DB é mais prevalente no sexo masculino, principalmente entre a terceira e quarta décadas de vida ${ }^{2}$.

A forma vascular da DB (DB vascular) acomete até um terço dos pacientes, sendo o envolvimento do sistema venoso mais comum que o do arterial, manifestando-se principalmente por trombose venosa profunda de membros inferiores. A DB vascular pode envolver também pequenas e grandes veias e artérias da circulação pulmonar, que são focos comuns de desenvolvimento de aneurismas, além de outras estruturas pulmonares, como parênquima e pleura. A prevalência do envolvimento pulmonar na DB varia de $1 \mathrm{a} 8 \%$, podendo se manifestar clinicamente com hemorragia alveolar, derrame pleural, embolia e hipertensão pulmonar, sendo a rotura local de aneurismas uma das principais causas de morte nos pacientes portadores da $\mathrm{DB}^{3,4}$.

A DB com acometimento do sistema nervoso central (neuro Behçet) pode se manifestar como tromboses de seios venosos, aneurismas, paralisia de nervos cranianos, sintomas neuropsiquiátricos e doença parenquimatosa, o que representa cerca de $80 \%$ dos casos, secundária a vasculites de pequenos vasos que atinge o tronco, hemisférios cerebrais e medula espinal ${ }^{1,2}$. O acometimento gastrointestinal na DB (gastro Behçet) é semelhante ao das doenças inflamatórias gastrointestinais, com manifestações como dor abdominal, diarreia e hemorragia digestiva ${ }^{2}$. As lesões cardíacas incluem pericardite, trombose, infarto agudo do miocárdio e fibrose endomiocárdica. Caracteristicamente, a DB acomete também o olho, causando uveíte posterior, trombose vascular e neurite óptica ${ }^{2}$.

O diagnóstico da DB é clínico. Em 1990, um conjunto de critérios de diagnóstico foi sugerido pelo International Study Group (ISG) ${ }^{5}$. Esses critérios incluem, além do critério obrigatório de úlceras orais recorrentes (pelo menos três episódios em 12 meses), os seguintes: lesões aftosas genitais recorrentes; lesões oculares como uveíte anterior ou posterior; manifestações cutâneas como eritema nodoso, pseudofoliculite, lesões papulopustulares ou nódulos acneiformes; e o teste de patergia positivo ${ }^{5}$. Tais critérios foram revisados e, em 2006, deram origem ao International Criteria Behçet Disease (ICBD), que incluiu as manifestações vasculares entre os critérios da $\mathrm{DB}^{2}$.

O chamado Behçet's Disease Current Activity Form (BDCAF) é um formulário com diversas questões apresentadas para entrevista do paciente com DB já diagnosticada, para caracterização da atividade da doença. Posteriormente, um modelo simplificado desse protocolo chamado de Behçet's Disease Activity Index (BDAI) foi apresentado pela Sociedade Internacional da DB. Esse formulário tem a finalidade de avaliar a presença de diferentes manifestações da DB nas 4 semanas anteriores à entrevista, que incluem diferentes manifestações clínicas, como: cefaleia, úlceras orais e genitais, eritema nodoso, pústulas, artralgia, artrite, dor abdominal/náuseas/vômitos, sangramento digestivo, sintomas oculares, comprometimento do sistema nervoso central e comprometimento de grandes vasos ${ }^{6}$. Assim, produz-se um índice, que varia de zero a 12, para avaliar a atividade da doença ${ }^{6}$.

A história natural da doença é composta por períodos de exacerbações e remissões, e sua patogênese é ainda incerta, mas há evidências que tendem para um mesmo caminho: um processo imunopatológico anormal é desencadeado, envolvendo diversos gatilhos, como infecção, por exemplo, associados à predisposição genética do alelo HLA-B512. Esses gatilhos ativam o sistema imunológico e iniciam o desenvolvimento da $\mathrm{DB}$ e, na sequência, iniciam a produção de inúmeras citocinas capazes de gerar células reativas responsáveis pelas lesões vasculares?

\section{MÉTODOS}

Trata-se de uma pesquisa definida como revisão bibliográfica de literatura. $\mathrm{O}$ estudo foi realizado por meio de artigos on-line, nacionais e internacionais e na língua inglesa, cujo corte temporal foi o período entre 2000 e 2020. Os artigos foram retirados das seguintes bases de dados: SciELO e PubMed. Utilizaram-se os seguintes descritores: "doença de Behçet", "patogenia da doença de Behçet" e "gene HLA-B51". Os critérios de inclusão foram textos completos e disponíveis, publicados nacionalmente e internacionalmente no período determinado.

\section{RESULTADOS}

Inicialmente, foram encontradas 12.687 referências nas bases de dados. Após o corte temporal de publicação, do ano 2000 até 2020, foram excluídos 4.435 artigos. Também foi um critério de exclusão 
artigos que não fossem de revisão, revisão sistemática, metanálise ou ensaio clínico, somando 6.902 estudos, e aqueles excluídos pelo título, totalizando 1.406. Foram selecionados, então, 106 artigos e eliminados aqueles em duplicidade. Os resumos dos 106 artigos foram lidos, sendo 19 deles excluídos por duplicidade e somente alguns enquadrados nos critérios de inclusão. Os demais (66) não abordavam o processo imunopatológico da DB ou acometimento vascular. Ao final, apenas 21 trabalhos foram considerados elegíveis por englobar o escopo de estudo do presente artigo, como resume a Figura 1.

As metanálises utilizadas foram a de Zhang et al. ${ }^{8}$ e a de Maldini et al. ${ }^{9}$. A primeira incluiu um total de 16 artigos, envolvendo 1.708 pacientes com DB e 1.910 controles saudáveis, com o objetivo de avaliar a associação entre polimorfismos do gene do fator de necrose tumoral (TNF), uma citocina pró-inflamatória na patogênese de vários distúrbios inflamatórios, incluindo a DB. Já a segunda selecionou 78 artigos que avaliaram de forma abrangente as relações entre as características clínicas da DB e o gene HLA-B51.

A revisão sistemática de Deng et al. ${ }^{10}$ abordou os vários fatores genéticos que podem contribuir para a patogênese da $\mathrm{DB}$ e os genes envolvidos na ativação e regulação imunológica. O estudo de Ahn et al. ${ }^{11}$ foi prospectivo, experimental e de caso-controle, que

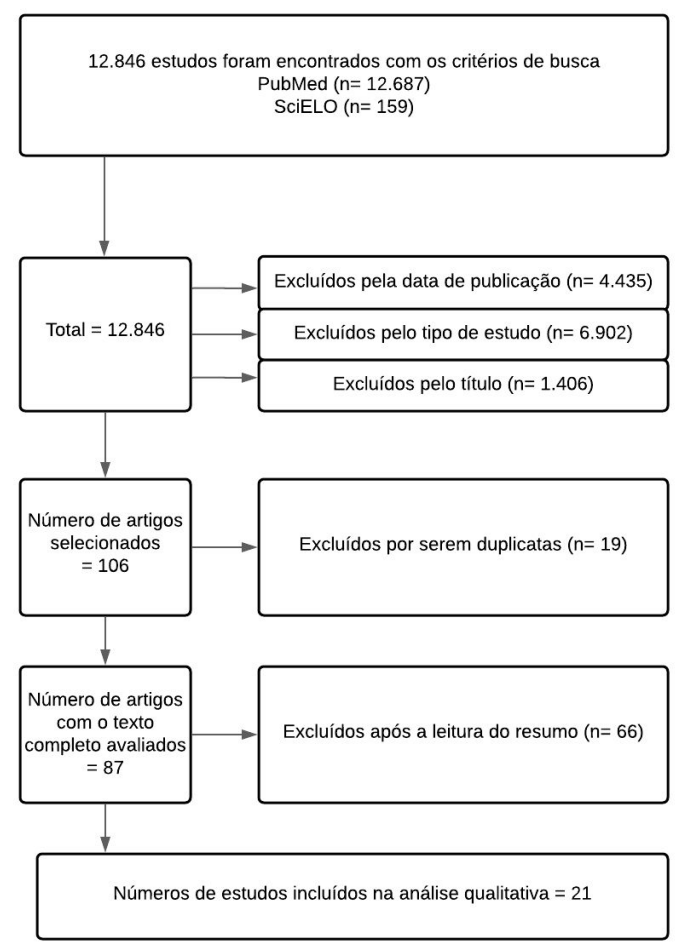

Figura 1. Fluxograma de seleção de artigos. determinou o perfil de citocinas no humor aquoso e no sangue periférico de pacientes com uveíte portadores da DB.

Outros artigos incluíram estudos retrospectivos (Alibaz-Oner et al. ${ }^{12}$ e Kechida et al. ${ }^{13}$ ) e estudos comparativos (Eksioglu-Demiralp et al. ${ }^{14}$, Borhani Haghighi et al. ${ }^{15}$ e Musabak et al. ${ }^{16}$ ), conforme detalhado na Tabela 1. Os demais artigos utilizados foram artigos de revisão que se enquadraram nos critérios de inclusão e foram considerados relevantes no tema do presente estudo ao abordarem aspectos introdutórios da doença, diferentes manifestações clínicas e aspectos imunopatológicos.

\section{DISCUSSÃO}

ADB é uma vasculite sistêmica caracterizada por aftas orais dolorosas e recorrentes, úlceras genitais, lesões oculares e cutâneas (Tabela 2). O envolvimento vascular é observado em até um terço dos casos, podendo acometer vasos de todos os tamanhos, tanto do sistema arterial quanto do venoso, levando a oclusões e/ou formação de aneurismas ${ }^{12}$. Os achados anatomopatológicos mais observados são infiltrados de linfócitos e neutrófilos, que resultam em lesão do endotélio vascular.

Entre os artigos de base desta revisão, houve uma notável heterogeneidade em relação às causas da DB. A etiologia ainda é incerta, porém certamente multifatorial, mas pode ser classificada em três principais e mais relevantes causas: genética, inflamatória e infecciosa. Esses três fatores inter-relacionados estão demonstrados na Figura 2, através do mecanismo imunopatológico da DB.

\section{Fator genético}

Desde os primeiros relatos da possível relação do alelo HLA-B51 com a DB, embora essa associação ainda se mantenha indefinida, houve uma série de estudos acerca do fato ${ }^{2}$. Muitos deles relataram prevalência mais alta do alelo HLA-B51 em pacientes portadores de $\mathrm{DB}$, além da associação com expressões mais complexas e graves das manifestações clínicas e desfechos piores do envolvimento ocular ou neurológico ${ }^{10}$. Ademais, foi demonstrado que o alelo HLA-B51 predomina em homens e está associado a prevalências moderadamente mais altas de úlceras genitais, manifestações oculares e cutâneas, além de alterações na coagulação e no endotélio, aumentando o risco de desenvolvimento de aneurismas e eventos trombóticos ${ }^{9,12}$.

Um estudo retrospectivo de 2004 a 2016 do Departamento de Medicina Interna e Endocrinologia do Hospital Universitário Fattouma Bourguiba, na Tunísia, mostrou a presença do alelo em 15,92\% dos 
Tabela 1. Estudos utilizados na revisão.

\begin{tabular}{|c|c|c|c|}
\hline Trabalho & Objetivo & Detalhes do Estudo & $\begin{array}{l}\text { Grau de } \\
\text { evidência } \\
\text { (Oxford) }\end{array}$ \\
\hline Alibaz-Oner et al. ${ }^{12}$ & $\begin{array}{l}\text { Investigar as abordagens } \\
\text { terapêuticas durante o } \\
\text { evento inicial e recidivas. }\end{array}$ & $\begin{array}{l}\text { Foram incluídos pacientes com DB ( } n=936 \text {, idade média: } \\
37,6 \pm 10,8 \text { ), classificados de acordo com os critérios do ISG } \\
\text { de } 15 \text { centros de reumatologia da Turquia }\end{array}$ & $2 \mathrm{C}$ \\
\hline Kechida et al. ${ }^{13}$ & $\begin{array}{l}\text { Descrever as características } \\
\text { clínicas da DB com o } \\
\text { envolvimento cardíaco e } \\
\text { vascular. }\end{array}$ & $\begin{array}{l}\text { Foram analisados } 213 \text { prontuários de todos os pacientes } \\
\text { com DB acompanhados entre janeiro de } 2004 \text { e maio } \\
\text { de } 2016 \text { no Departamento de Medicina Interna e que } \\
\text { preencheram os critérios do ISGBD. }\end{array}$ & $3 B$ \\
\hline Eksioglu-Demiralp et $\mathrm{al}^{4}$. & $\begin{array}{l}\text { Esclarecer o papel dos } \\
\text { neutrófilos na patogênese } \\
\text { da DB. }\end{array}$ & $\begin{array}{l}\text { A ativação de neutrófilos foi investigada em pacientes com } \\
\text { DB por métodos de citometria de fluxo. }\end{array}$ & $3 B$ \\
\hline Borhani Haghighi et al. ${ }^{15}$ & $\begin{array}{l}\text { Analisar perfis de citocinas } \\
\text { no LCR de pacientes com } \\
\text { doença neuro Behçet e } \\
\text { meningite viral. }\end{array}$ & $\begin{array}{l}\text { IL- }-6,8,10 \text {, fator de necrose tumoral- } \alpha \text { e interferon- } \gamma \\
\text { foram medidos no } \mathrm{LCR} \text { usando o método de ensaio } \\
\text { imunoenzimático. }\end{array}$ & $3 B$ \\
\hline Musabak et al. ${ }^{16}$ & $\begin{array}{l}\text { Investigar a relação dos } \\
\text { níveis séricos de IL-18 com } \\
\text { a atividade da doença e } \\
\text { as apresentações clínicas } \\
\text { da DB. }\end{array}$ & $\begin{array}{l}\text { Sessenta pacientes com DB e } 20 \text { controles saudáveis foram } \\
\text { incluídos no estudo. Os pacientes foram agrupados como } \\
\text { portadores de doença ativa ou inativa. }\end{array}$ & $3 B$ \\
\hline
\end{tabular}

DB = doença de Behçet; IL = interleucina; ISG = International Study Group; ISGBD = International Study Group for Behçet Disease; LCR = líquido cefalorraquidiano. 2C: Observação de resultados terapêuticos; 3B: Estudo caso-controle (Níveis de evidência científica segundo a classificação de Oxford Centre for Evidence-Based Medicine).

Tabela 2. Frequência de lesões clínicas na doença de Behçet.

\begin{tabular}{lc}
\hline & Frequência (\%) \\
\hline Lesões orais & 95 \\
Manifestação neurológica & $10-50$ \\
Manifestação gastrointestinal & $5-60$ \\
Manifestação vascular & $5-30$ \\
Manifestação cardíaca & $1-5$ \\
Manifestação pulmonar & $1-8$ \\
\hline
\end{tabular}

pacientes portadores da $\mathrm{DB}^{13}$. Neste mesmo estudo, realizado em 213 pacientes, também foram demonstrados dados importantes da DB como a presença das lesões orais em $98,6 \%$ dos pacientes em acompanhamento e $30 \%$ de acometimento vascular, sendo, portanto, importantes no diagnóstico da doença.

No entanto, os fatores genéticos não podem explicar totalmente a patogênese desta doença. A hipótese do alelo HLA-B51 associada à diversos gatilhos ambientais, como infecções bacterianas e virais, por exemplo, levando a uma exacerbação do processo imune e da resposta inflamatória parece ser mais sensata ${ }^{17}$.

Entretanto, a ausência do gene HLA-B51 em cerca de $80 \%$ dos pacientes, sugere que outros fatores também devem ser avaliados na gênese da $\mathrm{DB}^{10,18}$.

\section{Processo inflamatório e imunológico}

A resposta inflamatória e o processo imunológico são incertos e complexos e podem levar a alteração da cascata de coagulação e lesão endotelial, favorecendo a formação de trombos, êmbolos e aneurismas ${ }^{19}$. Recentemente, a imunopatogênese e o envolvimento das células imunes e adaptativas e citocinas na gênese da DB vêm sendo debatidas ${ }^{10}$.

As células natural killer (NK), as principais da imunidade inata, não apenas desempenham um papel citotóxico nas células infectadas, mas também regulam as funções de outras células do sistema imune secretoras de citocinas, dentre elas TNF- $\alpha$, interferon (IFN) $\gamma$ e $\alpha$ e interleucinas (IL) 2, 4 e $10^{7}$.

Os neutrófilos também desempenham um papel fundamental na resposta imune inata e caracterizam-se por serem a primeira linha de defesa contra doenças infecciosas. Estudos demonstraram que um dos principais mecanismos na patogênese da DB é a ativação de neutrófilos com aumento da quimiotaxia e da geração de radicais superóxido. Além disso, um estudo evidenciou que os neutrófilos nos pacientes com a DB exibem alta ativação intrínseca que pode estar associada à presença do gene HLA-B51 e estão envolvidos na infiltração perivascular das lesões da DB vascular ${ }^{14}$.

As respostas adaptativas são representadas na doença pelas células T de padrão de resposta Th 1 e 17, que desempenham um papel importante na patogênese da doença ${ }^{7}$. A resposta imune mediada por células Th1 desempenha um papel fundamental na patogênese da DB, e sua prevalência é significativamente maior em pacientes com DB ativa do que naqueles em pacientes com DB inativa ${ }^{7}$. A Tabela 3 sintetiza como 


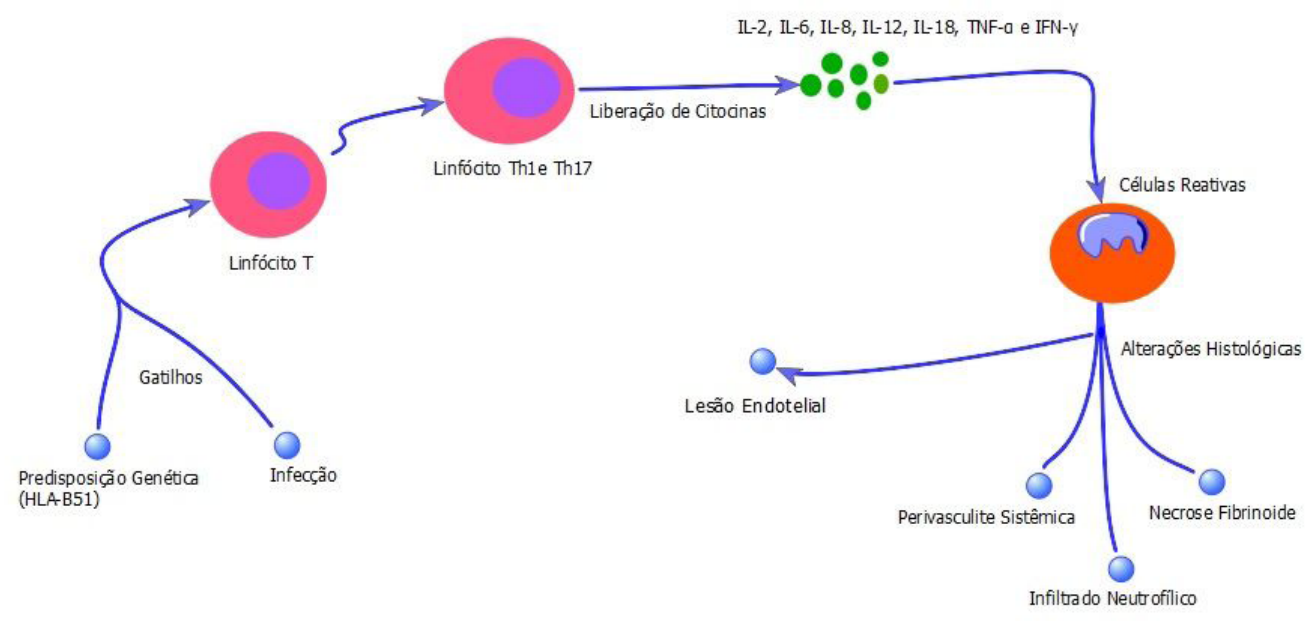

Figura 2. Diagrama do mecanismo imunopatológico da doença de Behçet - o alelo HLA-B51 associado às infecções medeiam a patogenia da doença de Behçet. Tais gatilhos ativam um sistema imunológico inadequadamente capazes de produzir citocinas e, consequentemente, células reativas, lesionando, por fim, os vasos. IL = interleucina; IFN = interferon; TNF = fator de necrose tumoral.

Tabela 3. Principais células envolvidas na patogênese da doença de Behçet.

\begin{tabular}{ll}
\hline Célula NK & $\begin{array}{l}\text { Nas doenças autoimunes, a frequência das células NK diminui e sua citotoxicidade é prejudicada. Alguns estudos } \\
\text { demonstraram que o número de células NK em pacientes com DB diminuíram, comprometendo a imunidade. } \\
\text { Neutrófilos }\end{array}$ \\
Neutrófilos podem danificar células e tecidos hospedeiros, sendo, assim, o fator desencadeante da inflamação, que, \\
por sua vez, desencadeia a resposta imune.
\end{tabular}
$\mathrm{DB}=$ doença de Behçet; IFN = interferon; NK = natural killer.

essas células atuam na patogênese da doença, além do papel das citocinas envolvidas.

\section{Infecções}

Infecções bacterianas ou virais podem servir de gatilhos para o desenvolvimento da doença em indivíduos portadores do alelo HLA-B51 ${ }^{12}$. Os microrganismos mais associados à DB são o herpes vírus simplex ou Streptococcus ssp, porém também há descrições de outros, como parvovírus B19, Helicobacter pylori, citomegalovírus, Epstein-Barr vírus, herpes zoster vírus e Staphylococcus aureus ${ }^{2,7}$.

Essas infecções estão associadas à inflamação sistêmica que, em indivíduos geneticamente suscetíveis, pode desencadear uma resposta imune inadequada e exacerbada e provocar lesão endotelial. Esse processo é demonstrado na Figura 2.

\section{O papel das citocinas inflamatórias}

A imunopatogênese da DB foi amplamente estudada e várias células do sistema imunológico e citocinas podem estar envolvidas, sobretudo as pró-inflamatórias, que aceleram o prejuízo da função endotelial ${ }^{7}$. Um estudo evidenciou que muitas dessas citocinas inflamatórias foram encontradas, de forma aumentada, em soro de pacientes com DB, e, por causa dessas evidências, os antagonistas de TNF- $\alpha$ e IFN- $\alpha$ demonstraram boa eficácia e são os agentes usados no tratamento da $\mathrm{DB}^{7}$.

$\mathrm{Na}$ Figura 2, primeiramente, observa-se uma associação da doença com o alelo HLA-B51, que demonstra risco maior para a DB. Os agentes infecciosos também podem servir de gatilho para uma resposta imunológica anormal. Ambos podem desencadear a hiper-reatividade das células $\mathrm{T}$ e neutrófilos ${ }^{14}$. Os linfócitos T respondem a infecções virais e bacterianas, tendendo, assim, para um padrão de resposta Th1 e Th17, que produzem mediadores pró-inflamatórios, como IL-2, IL-6, IL-8, IL-12, IL- 18, IL-21, TNF- $\alpha$ e IFN- $\gamma^{7}$. Vale ressaltar como algumas delas se relacionam com a DB:

IL-6: a produção excessiva de IL-6 está relacionada a doenças inflamatórias autoimunes e crônicas. Foi relatado aumento de IL-6 no líquido cefalorraquidiano em pacientes com envolvimento neurológico na $\mathrm{DB}^{15}$. 
IL-18: desempenha um papel importante na resposta imune das células Th1. As funções da IL-18 são promover a produção de IFN- $\gamma$ ativando células NK e induzir a atividade citotóxica. Os níveis de IL-18 foram mais altos em todos os subgrupos de pacientes quando comparados aos controles saudáveis, demonstrando a correlação dessa citocina com a atividade da doença ${ }^{16}$.

IFN- $\gamma$ : as células Th1 que produzem IFN- $\gamma$ ativam macrófagos e estão associadas a doenças autoimunes, incluindo a $\mathrm{DB}^{7}$. Em um estudo que avaliou o papel das citocinas na uveíte da $\mathrm{DB}$, foi relatado que o nível de IFN- $\gamma$ no humor aquoso dos pacientes com DB foi significativamente maior do que nos pacientes com $\mathrm{DB}$, porém sem uveíte ${ }^{11}$.

TNF- $\alpha$ : desempenha um papel fundamental na indução e manutenção da inflamação na resposta autoimune, além de estar envolvido em vários processos fisiológicos e patológicos, como o início da inflamação, imunorregulação e proliferação celular ${ }^{8}$. Nas doenças inflamatórias, o TNF- $\alpha$ é produzido principalmente por macrófagos, células $\mathrm{T}$, células $B$, neutrófilos, células NK e células endoteliais. Os níveis séricos de TNF- $\alpha$ estão aumentados em pacientes com DB ativa?

IL-21: é uma citocina da família das IL-2, que promove expansão de células $\mathrm{T} \mathrm{CD} 8^{+}$efetoras e pode ativar células $\mathrm{NK}^{7}$. Há evidências do papel crítico da IL-21 na condução de lesões inflamatórias na DB, promovendo Th17 efetores e suprimindo as células T reguladoras, representando um alvo promissor para uma nova terapia na $\mathrm{DB}^{20}$.

\section{Acometimento vascular na DB}

A vasculite na DB é predominantemente neutrofílica, acometendo todas as camadas do vaso e vasa vasorum, podendo apresentar espessamento fibroso e infiltrado inflamatório inespecífico em sua fase tardia ${ }^{5}$. Associado à vasculite, há também presença de hipercoagulabilidade, com formação excessiva de trombina, diminuição de fibrinólise, hiperatividade plaquetária e formação dos complexos plaqueta/ neutrófilos ${ }^{2}$. Todas essas alterações podem favorecer a formação de trombose. O processo inflamatório na parede do vaso pode causar destruição das fibras elásticas, levando à necrose transmural de parede das grandes artérias musculares, com consequente formação de aneurismas ${ }^{5}$.

\section{Tratamento}

Apesar das opções de terapias tradicionais com uso de glicocorticoides e imunossupressores, há poucas evidências quanto à eficiência desses tratamentos na $\mathrm{DB}^{1}$. Os antagonistas do TNF- $\alpha$ e IFN têm demonstrado boa eficácia e são os agentes de primeira linha usados para melhorar o prognóstico da DB. O tratamento dos pacientes com antagonistas do TNF- $\alpha$ (Infliximabe, Etanercepte, Adalimumabe) se baseia no controle da resposta inflamatória, eficiente em manifestações graves e refratárias da $\mathrm{DB}^{7}$. A terapia com IFN, sobretudo o INF- $\alpha$, tem demonstrado benefícios no tratamento habitual da doença, com atividades antiviral, antitumoral e imunomoduladora eficazes no manejo da $\mathrm{DB}^{1}$. Porém, ainda há escassez de dados sobre essas abordagens terapêuticas ideais e falta de marcadores laboratoriais informativos para monitorar a progressão da doença ${ }^{21}$.

\section{CONCLUSÃO}

A patogênese da DB ainda não é bem esclarecida. Entretanto, estudos têm demonstrado que alguns gatilhos, como infecções bacterianas e virais, e predisposição genética (presença do alelo HLA-B51) podem iniciar e interferir no desenvolvimento da DB, pela ativação exacerbada do sistema imunológico, resultando da produção de inúmeras citocinas capazes de gerar células reativas responsáveis pelas lesões nos vasos e hipercoagulabilidade. Contudo, estudos mais detalhados são necessários para elucidar melhor os mecanismos envolvidos no processo imunopatológico da DB.

\section{REFERÊNCIAS}

1. Neves FS, Moraes JC, Gonçalves CR. Síndrome de Behçet: à procura de evidências. Rev Bras Reumatol. 2006;46(Supl. 1):21-9. http:// dx.doi.org/10.1590/S0482-50042006000700005.

2. Ferrão C, Almeida I, Marinho A, Vasconcelos C, Correia JA. A nossa regra de ouro na doença de Behçet: tratar a manifestação clínica. Arq Med. 2015;29(3):75-9.

3. Erkan F, Gül A, Tasali E. Pulmonary manifestations of Behçet's disease. Thorax. 2001;56(7):572-8. http://dx.doi.org/10.1136/ thorax.56.7.572. PMid:11413359.

4. Yuan S-M. Pulmonary artery aneurysms in Behçet disease. J Vasc Bras. 2014;13(3):217-28. http://dx.doi.org/10.1590/jvb.2014.041.

5. Seyahi E, Yurdakul S. Behçet's Syndrome and thrombosis. Mediterr J Hematol Infect Dis. 2011;3(1):e2011026. http://dx.doi.org/10.4084/ mjhid.2011.026. PMid:21869912.

6. Neves FS, Caldas CAM, Medeiros DM, Moraes JC, Gonçalves CR. Adaptação transcultural da versão simplificada (s) do Behçet's Disease Current Activity Form (BDCAF) e comparação do desempenho das versões brasileiras dos dois instrumentos de avaliação da atividade da Doença de Behçet: BR-BDCAF e BRBDCAF(s). Rev Bras Reumatol. 2009;49(1):20-31. http://dx.doi. org/10.1590/S0482-50042009000100003.

7. Tong B, Liu X, Xiao J, Su G. Immunopathogenesis of Behcet's disease. Front Immunol. 2019;10:665. http://dx.doi.org/10.3389/ fimmu.2019.00665. PMid:30984205.

8. Zhang $M, X u$ WD, Wen PF, et al. Polymorphisms in the tumor necrosis factor gene and susceptibility to Behcet's disease: an updated meta-analysis. Mol Vis. 2013;19:1913-24. PMid:24049437. 
9. Maldini C, Lavalley MP, Cheminant M, de Menthon M, Mahr A Relationships of HLA-B51 or B5 genotype with Behcet's disease clinical characteristics: systematic review and meta-analyses of observational studies. Rheumatology (Oxford). 2012;51(5):887-900. http://dx.doi.org/10.1093/rheumatology/ker428. PMid:22240504.

10. Deng $Y$, Zhu W, Zhou X. Immune regulatory genes are major genetic factors to behcet disease: systematic review. Open Rheumatol J. 2018;12(1):70-85. http://dx.doi.org/10.2174/1874312901812010 070. PMid:30069262.

11. Ahn JK, Yu HG, Chung H, Park YG. Intraocular Cytokine Environment in Active Behçet Uveitis. Am J Ophthalmol. 2006;142(3):429-34. http://dx.doi.org/10.1016/j.ajo.2006.04.016. PMid:16935587.

12. Alibaz-Oner F, Karadeniz A, Ylmaz S, et al. Behçet disease with vascular involvement: effects of different therapeutic regimens on the incidence of new relapses. Medicine. 2015;94(6):e494. http:// dx.doi.org/10.1097/MD.0000000000000494. PMid:25674739.

13. Kechida M, Salah S, Kahloun R, Klii R, Hammami S, Khochtali I. Cardiac and vascular complications of Behçet disease in the Tunisian context: clinical characteristics and predictive factors. Adv Rheumatol. 2018;58(1):32. http://dx.doi.org/10.1186/s42358018-0032-x. PMid:30657088.

14. Eksioglu-Demiralp E, Direskeneli H, Kibaroglu A, Yavuz S, Ergun T, Akoglu T. Neutrophil activation in Behçet's disease. Clin Exp Rheumatol. 2001;19(5, Supl 24):S19-24. PMid:11760393.

15. Borhani Haghighi A, Ittehadi H, Nikseresht AR, et al. CSF levels of cytokines in neuro-Behçet's disease. Clin Neurol Neurosurg. 2009;111(6):507-10. http://dx.doi.org/10.1016/j.clineuro.2009.02.001. PMid:19303205.

16. Musabak U, Pay S, Erdem H, et al. Serum interleukin-18 levels in patients with Behçet's disease: is its expression associated with disease activity or clinical presentations? rheumatol int. 2006;26(6):545-50. http://dx.doi.org/10.1007/s00296-005-0029-8. PMid:16205927.

17. Deuter CM, Kotter I, Wallace GR, Murray PI, Stubiger N, Zierhut M. Behcet's disease: ocular effects and treatment. Prog Retin Eye Res. 2008;27(1):111-36. http://dx.doi.org/10.1016/j.preteyeres.2007.09.002. PMid:18035584.
18. Yazici H, Seyahi E, Hatemi G, Yazici Y. Behçet syndrome: a contemporary view. Nat Rev Rheumatol. 2018;14(2):107-19. http:// dx.doi.org/10.1038/nrrheum.2017.208. PMid:29296024.

19. Owlia MB, Mehrpoor G. Behcet's disease: new concepts in cardiovascular involvements and future direction for treatment. ISRN Pharmacol. 2012;2012:760484. http://dx.doi.org/10.5402/2012/760484. PMid:22530146.

20. Geri G, Terrier B, Rosenzwajg M, et al. Critical role of IL-21 in modulating $\mathrm{TH} 17$ and regulatory T cells in Behçet disease. J Allergy Clin Immunol. 2011;128(3):655-64. http://dx.doi.org/10.1016/j. jaci.2011.05.029. PMid:21724243.

21. Ambrose N, Haskard D. Differential diagnosis and management of Behçet syndrome. Nat Rev Rheumatol. 2013;9(2):79-89. http:// dx.doi.org/10.1038/nrrheum.2012.156. PMid:23007742.

Correspondência
Cristiane Tefé-Silva
Av. Luis Eduardo de Toledo Prado, 847, Apartamento 13, Torre 1,
Reserva do Golf - Vila do Golf
CEP 14027-250, Ribeirão Preto (SP), Brasil
Tel.: (16) 99173-1522/(16) 3603-6672
E-mail: cris_tefe@ @hotmail.com
Informações sobre os autores
RMV, MLNC, MPHG, BMS e GIS - Graduandas de Medicina, Centro
Universitário Barão de Mauá.
KFZ e CTS - Docentes, Centro Universitário Barão de Mauá;
Contribuições dos autores
Concepção e desenho do estudo: RMV, MPHG, CTS
Análise e interpretação dos dados: RMV, GIS
Coleta de dados: RMV, MLNC, MPHG, BMS, GIS
Redação do artigo: RMV, MLNC
Revisão crítica do texto: KFZ, CTS
Aprovação final do artigo: KFZ, CTS
Análise estatística: N/A.
*Todos os autores leram e aprovaram a versão final submetida ao J
Vasc Bras.

